\title{
Behavioral contrast in multiple schedules with equal reinforcement rates ${ }^{1}$
}

\author{
AARON J. BROWNSTEIN and \\ CRIGHTON NEWSOM, The University of \\ North Carolina at Greensboro, Greensboro, \\ N. C. 27412
}

After responding was maintained on multiple fixed-interval schedules of reinforcement, a cuing procedure was added to one component. Cuing was accomplished by iluminating a lamp just prior to reinforcement availability. This procedure produced positive behavioral contrast. The rate of responding in the cued component decreased, and the rate of responding in the uncued component increased. When the cue was removed, negative behavioral contrast occurred. Rate of responding in the component from which the cue had been removed increased, and rate of responding in the other component decreased. Throughout the experiment, rates of reinforcement in both components were held constant.

If opposing rate changes in the two components of a multiple schedule accompany the direct manipulation of the rate of responding in one of the components, behavioral contrast is said to occur. Contrast is reliably produced when the rate of responding is altered by a change in the rate of reinforcement (Reynolds, 1961a, b). .Reynolds (1961b) has proposed the relative rate of reinforcement as a primary determinant of contrast. For components of equal duration, relative rate of reinforcement in a given component is equal to the percentage of the total reinforcement provided in that component. If the rate of reinforcement in one component of a multiple schedule is held constant, the relative rate of reinforcement in that component varies inversely with changes in the rate of reinforcement in the other component. If rate of responding is determined in part by relative reinforcement rate, the contrast effect would be expected when reinforcement rate is varied in one component.

Three recent studies have reported positive behavioral contrast in multiple schedules in which no increase in relative reinforcement rate was present in the component in which response rate increased. Reynolds \& Limpo (1968) stabilized responding in a two-component multiple schedule in which low rates of responding were reinforced (DRL schedule). Responding in one component was supressed by adding an interresponse time (IRT) clock. The IRT clock consisted of a series of lights that were sequentially illuminated at successive 5-sec intervals after the preceding response. Responding in the component not containing the clock increased. The rate of reinforcement in the component not containing the clock decreased, while rate of reinforcement in the component containing the clock increased. Terrace (1968) maintained responding on an isolated variable-interval (VI) schedule of reinforcement. The schedule was then shifted to a multiple schedule in which the VI was kept intact as one component, and a DRL schedule prevailed in the other component. DRL values were chosen such that obtained reinforcement rates in the DRL component were equal to those obtained in the VI component. Rate of responding in the VI component increased relative to the rate in the VI in isolation in three of six birds. Weisman (1969) maintained responding on multiple VI schedules of reinforcement. One component was switched to DRL, with values chosen so that obtained reinforcement rates in the DRL and VI components were equal. Rate of responding in the DRL component decreased, and rate of responding in the VI component increased.

Each of these studies employed a DRL schedule in the component in which responding was suppressed. In two of the studies, the DRL was introduced to suppress responding. In the third, a clock was added to an already present DRL component. The present experiment is concerned with contrast effects produced by response suppression in multiple fixed-interval (FI) schedules of reinforcement, in which reinforcement rates are unaltered by the reduction in rate of responding. Responding in one component of multiple FI schedules of reinforcement was suppressed by an added cue. The cuing procedure consisted of illuminating a lamp $3 \mathrm{sec}$ prior to the expiration of the FI. The suppression technique is similar to that used by Reynolds \& Limpo (1968) but avoids the large changes in reinforcement rates produced by response suppression in a DRL schedule.

\section{SUBJECTS AND APPARATUS}

Four adult Silver King pigeons were maintained at $80 \%$ of their free-feeding body weights throughout the experiment. All four Ss were experimentally naive at the start of this study, and all were hand-shaped to a key-peck response within one session.

A standard Lehigh Valley Electronics operant-conditioning chamber for pigeons was used in this experiment. The response key could be transilluminated with red or green light, and a relay feedback click accompanied each response. A jewel light receptacle was mounted on the front wall, $11 / 2$ in. to the left of and 2 in. above the response key. A Westinghouse auto bulb, No. W-304, was used with a 1-in.-diam amber jewel; this served as the "cue" light. During reinforcement ( $3 \mathrm{sec}$ access to grain), the chamber was dark and the grain magazine was illuminated. White noise and the noise of the ventilating fan masked extraneous sounds. Standard electromechanical programming equipment was employed; data were recorded on digital counters and on a cumulative recorder.

\section{PROCEDURE}

Each daily session consisted of four cycles of a two-component multiple fixed-interval 2-min schedule of reinforcement. A cycle comprised 10 fixed intervals. The response key was transilluminated with red light during the first five fixed intervals (the red component) and with green light during the last five fixed intervals (the green component). This schedule of reinforcement was in effect for 66 sessions of preliminary training. Then the scheduled reinforcements in the red component were cued in the following manner: The amber light cue was illuminated $3 \mathrm{sec}$ prior to the expiration of each of the five 2-min intervals; during the time the cue light was illuminated, the red key light and the houselight remained on. This schedule of reinforcement, in which the reinforcements scheduled in the red component were always cued and those in the green component were never cued, was in effect for 50 sessions. During the next 10 sessions, the cue was omitted. Then the cue in the red component was reinstated for 50 additional sessions.

\section{RESULTS}

The data for each bird were treated in the following manner. The mean overall rate of responding in each component of the multiple schedule for the five sessions (62-66) preceding the introduction of the cue was obtained. This block of sessions was designated as Period 0 . Mean overall rates were also obtained for Sessions 72-76, $82-86,92-96$, and $112-116$. The successive blocks were respectively designated Periods 1, 2, 3, and 5. Data for Sessions 102-106 (Period 4) were lost because of a recording error. Overall rates of responding in each period were normalized with respect to 


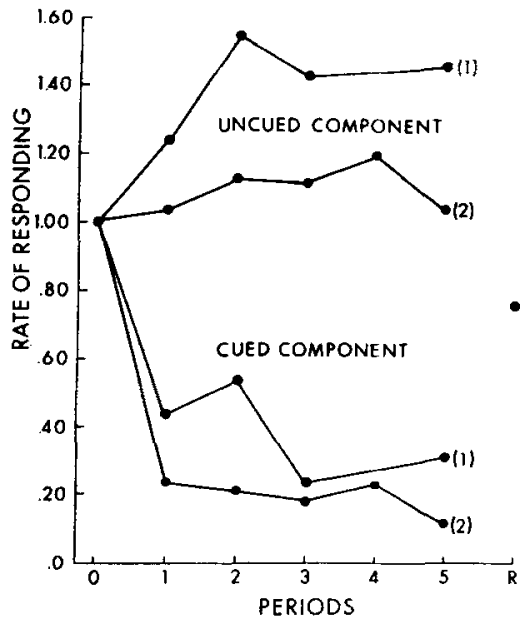

Fig. 1. Average response rates per period of five sessions in cued and uncued components. Rates in Periods $0-5$ are normalized with respect to Period 0 . (See text for further details.)

performance during Period 0 . The average for all birds was then obtained. The results of this analysis are shown in Fig. 1 by the curves labeled uncued component (1) and cued component (1). The cuing procedure effectively reduced the rate of responding in the component with the added cue. The procedure was effective for each bird. Of major interest is the increased response rate in the uncued component. The average function presented is representative of each bird. For each bird, rate of responding in each period, except for Period 0 , is greater than 1.00. Considerable variability in the magnitude of the increase in response rate was found. Such variability is not uncommon in contrast data. Peak increases ranged from $16 \%$ to $140 \%$ over the four birds. The median peak increase was $35 \%$.

The point in Fig. 1 with abscissa $R$ is based on performance during Sessions 122-126 (Sessions 6-10 after the cue was removed). Performance during this period was normalized with respect to
Period 5, the last five sessions containing the cue. The ordinate for this point is the average for all birds during the component that had always been uncued. The decrease in average response rate was found in each of the birds. A corresponding increase in response rate during this period in the previously cued component cannot be presented easily without distorting the scale. The average increase was $500 \%$.

The curves labeled uncued component (2) and cued component (2) were obtained following the procedures described above. Period 0 was based on the last five sessions preceding the reintroduction of the cue (Period R in the above). Periods 1 through 5 were respectively based on Sessions 6-10, $16-20,26-30,36-40$, and $46-50$ following the reintroduction of the cue.

The data treatment described above with respect to overall rate of responding was also carricd out on postreinforcement pause and on running-rate measures. The time base for running rate excluded the time between the start of the interval and the first response in the interval, i.e., the post $r$ e inforcement pause. Postreinforcement pause increased and running rate decreased for all birds in the cued component. The overall rate increases during the uncued component, reported above, were modulated by an increase in running rate and a decrease in postreinforcement pause for two of the birds, only an increase in running rate for one of the birds, and only a decrease in postreinforcement pause for one of the birds. For the two birds where both measures changed, a greater relative change occurred in the rate measure. Additionally, the bird showing the least relative increase in overall rate was the one for which only postreinforcement pause decreased.

\section{DISCUSSION}

The present data show clear evidence of the occurrence of positive and negative behavioral contrast. The rate of responding in the green component of the multiple s chedule-the unaltered component-increased when the rate of responding in the red component was decreased by adding the cue. When the cue was removed, rate of responding in the red component increased, and rate of responding in the green component decreased. Positive and negative behavioral contrast occurred in the absence of any change in rate or relative rate of reinforcement in either component.

The results of this study are consistent with the view that a reduction in the rate of responding may generate behavioral contrast (Reynolds \& Limpo, 1968; Terrace, 1963a,b, 1968). The results extend the generality of this notion by demonstrating the effect with a different procedure employed to reduce response rate. However, as is true with other procedures, the amount of contrast was not correlated with the degree of suppression. This absence of a correlation was true within the performance of individual birds as well as across birds.

\section{REFERENCES}

REYNOLDS, G. S. Behavioral contrast. Journal of the Experimental Analysis of Behavior, 1961a, 4, 57-71.

REYNOLDS, G. S. Relativity of response rate and reinforcement frequency in a multiple schedule. Journal of the Experimental Analysis of Behavior, 1961b, 4, 179-184.

REYNOLDS, G. S., \& LIMPO, A. J. On some causes of behavioral contrast. Journal of the Experimental Analysis of Behavior, 1968, 11, 543-547.

TERRACE, H. S. Discrimination training with and without "errors." Journal of the Experimental Analysis of Behavior, 1963a, 6, 1-27.

TERRACE, H. S. Errorless transfer of a discrimination across two continua. Journal of the Experimental Analysis of Behavior, 1963b, $6,223-232$.

TERRACE, H. S. Discrimination learning, the peak shift and behavioral contrast. Joumal of the Experimental Analysis of Behavior, 1968, 11, 727-741.

WEISMAN, R. G. Some determinants of inhibitory stimulus control. Journal of the Experimental Analysis of Behavior, 1969, 12, 443-450.

NOTE

1. This research was supported in part by a grant from the Research Council of the University of North Carolina at Greensboro. 